\title{
ARE WAVE FUNCTIONS UNIQUELY DETERMINED BY THEIR POSITION AND MOMENTUM DISTRIBUTIONS?
}

\author{
J. V. CORBETT and C. A. HURST
}

(Received 29 August 1977)

(Revised 29 November 1977)

\begin{abstract}
The problem of determining a square integrable function from both its modulus and the modulus of its Fourier transform is studied. It is shown that for a large class of real functions the function is uniquely determined from this data. We also construct fundamental subsets of functions that are not uniquely determined. In quantum mechanical language, bound states are uniquely determined by their position and momentum distributions but, in general, scattering states are not.
\end{abstract}

\section{Introduction}

\section{The Pauli problem}

In a footnote to his Handbuch der Physik article on the general principles of wave mechanics [4], Pauli raised the question of whether the wave function $\psi(q)$ was uniquely determined by the probability densities $|\psi(q)|^{2}$ and $|\psi(p)|^{2}$ in configuration and momentum space. In this article we show that there are fundamental subsets where the answer is no and there is a large and interesting class of functions $\phi(q) \in L^{2}(\mathbf{R})$ for which the answer is yes.

The question was motivated by the fact that if the wave function was uniquely determined by its position and momentum probability densities then we have, at least in principle, a method of determining the wave function from experiment. Nevertheless, we should point out that the mathematical problem is of interest in other contexts, such as control theory and crystallography. We will comment further on the physical meaning of this problem and our results in the final section.

The mathematical problem is that of determining the phase of a complex-valued square integrable function given both the amplitude of the function and the 
amplitude of its Fourier transform. We are particularly interested in the uniqueness of the solution to this problem. For example, assume that $\phi(q) \in L^{2}(\mathbf{R})$ and let $\hat{\phi}(p) \in L^{2}(\mathbf{R})$ be its Fourier transform, then given $|\phi(q)|$ and $|\hat{\phi}(p)|$ we have that $\phi(q)=|\phi(q)| e^{i \theta(q)}$ is a square integrable function with modulus $|\phi(q)|$ and with $|\hat{\phi}(p)|$ the modulus of its Fourier transform. The real-valued function $\theta(q)$ is the phase of $\phi(q)$. Is there any other square integrable function $\psi(q)$ with $|\psi(q)|=|\phi(q)|$ and $|\hat{\psi}(p)|=|\hat{\phi}(p)|$ ? This function $\psi(q)$ will be of the form $\psi(q)=|\phi(q)| e^{i \alpha(q)}$ for some real-valued function $\alpha(q)$. If we concentrate on the phase function $\alpha(q)$ it is clear that the set of permissible phase functions is given by the solutions of the following non-linear integral equation

$$
|\hat{\phi}(p)|^{2}=\frac{1}{2 \pi} \int_{-\infty}^{\infty} d q \int_{-\infty}^{\infty} d q^{\prime} K_{p}\left(q, q^{\prime}\right) e^{i\left[\alpha(q)-\alpha\left(q^{\prime}\right)\right]}
$$

with $K_{p}\left(q, q^{\prime}\right)=|\phi(q)|\left|\phi\left(q^{\prime}\right)\right| e^{i p\left(q-q^{\prime}\right)}$, where we have used the convention that

$$
\hat{\phi}(p)=\frac{2}{\sqrt{2 \pi}} \int_{-\infty}^{\infty} e^{i p q} \phi(q) d q .
$$

It is clear from equation (0) that if $\alpha(q)=\theta(q)+\beta$ for any real constant $\beta$ then $\alpha(q)$ is a solution of this equation. In the following we will ignore this trivial non-uniqueness and consider only the problem of non-uniqueness modulo an arbitrary constant phase.

The problem of uniqueness of the phase $\phi(q)$ depends strongly upon the given function $\alpha(q)$ and it is not clear how general results can be obtained from the equation (0). In fact we have had to forgo a direct attack on this non-linear integral equation and have used elementary methods to construct examples in which the Pauli data $\{|\phi(q)|,|\hat{\phi}(p)|\}$ is sufficient to determine $\phi(q)$ uniquely and examples in which it is not sufficient to uniquely define the wave function $\phi(q)$.

We adopt a terminology in which a square integrable function $\phi(q)$ is said to be Pauli unique if the Pauli data $\{|\phi(q)|,|\hat{\phi}(p)|\}$ obtained from it is only satisfied by the ray of functions that are constant multiples of $\phi(q)$ with constant multiplier of modulus one. If a function $\phi(q)$ is not Pauli unique we call any function $\psi(q)$ that satisfies the same Pauli data a Pauli partner of $\phi(q)$. Furthermore, it is often useful to think of the square summable function $\phi(q)$ as a state, that is a normalized positive linear functional over the vector space of operators spanned by the algebra of operators $\mathfrak{a}_{p}$ and $\mathfrak{a}_{q}$, where $\mathfrak{a}_{q}$ is the commutative algebra of functions of $q$ and $\mathfrak{a}_{p}$ is the commutative algebra of functions of $p$.

In Section 2 we give precise definitions of our terminology and obtain some general characteristics of the problem. In Sections 3 and 4 we restrict attention to the Hilbert space $L^{2}(\mathbf{R})$ and use elementary methods to construct examples of unique states and of non-unique states. In Section 3 we construct a two parameter 
family of fundamental subsets of $L^{2}(\mathbf{R})$ every member of which is Pauli non-unique. In Section 4 we show that there is a large class of real states that are Pauli unique and prove amongst other things that every bound state of any self-adjoint semibounded Hamiltonian of the form $p^{2}+V(q)$ is Pauli unique. In Section 5 the results of the previous two Sections are generalized to Hilbert spaces $L^{2}\left(\mathbf{R}^{n}\right)$, and the final Section discusses the physical significance of these results and their generalization.

\section{Notation and formulation of the problem}

We will adopt the formulation of quantum mechanics in which to every physical system there is associated a complex Hilbert space $\mathscr{H}$. Every normalized vector $\phi$ of $\mathscr{H}$ represents a possible state of the system, and two vectors $\phi$ and $\psi$ define the same state if they belong to the same ray, that is, if there exists a real number such that $\phi=e^{i \theta} \psi$. Furthermore, every physical quantity associated with the system is represented by a self-adjoint operator $A$, usually unbounded.

Assume that the system is a collection of $n$ scalar particles with masses $m_{i}$, $i=1, \ldots, n$, that are interacting in some way. Let the $n$ position coordinates be $\mathbf{q}_{1}, \ldots, \mathbf{q}_{n}$ and the $n$ momenta be $\mathbf{p}_{1}, \ldots, \mathbf{p}_{n}$. Let us further assume that the system is represented by the standard irreducible representation of the Heisenberg commutation relations,

and

$$
\left.\begin{array}{l}
{\left[\mathbf{q}_{i}, \mathbf{q}_{j}\right]=\left[\mathbf{p}_{i}, \mathbf{p}_{j}\right] \subset 0} \\
i\left[p_{j}, q_{k}\right] \subset h \delta_{i j} .
\end{array}\right\}
$$

The standard irreducible representation is, in fact, an equivalence class of representations characterized by the fact that the Heisenberg commutation relations are derived from an irreducible unitary representation of the Weyl group [5]. The two realizations of this representation that interest us here both have $\mathscr{H}=L^{2}\left(\mathbf{R}^{3 n}\right)$ and in the first, the $q$-representation, the vectors in $\mathscr{H}$ are equivalence classes of functions $\phi(q)=\phi\left(\mathbf{q}_{1}, \ldots, \mathbf{q}_{n}\right)$ in $L^{2}\left(\mathbf{R}^{3 n}\right)$ while in the second, the $p$-representation the vectors are equivalence classes of functions $\hat{\phi}(p)=\hat{\phi}\left(\mathbf{p}_{1}, \ldots, \mathbf{p}_{n}\right)$ belonging to $L^{2}\left(\mathbf{R}^{3 n}\right)$. The equivalence class alluded to above is given by the relation that two functions are equivalent if they only differ on a set of Lebesgue measure zero. These $q$ - and $p$-realizations of the Heisenberg commutation relations are related by Fourier transformations,

$$
\hat{\phi}(p)=F \phi=(2 \pi)^{-3 n / 2} \int e^{+i p q} \phi(q) d q,
$$

where the argument $q$ stands for $\left(\mathbf{q}_{1}, \ldots, \mathbf{q}_{n}\right), d q=d^{3} q_{1} \ldots d^{3} q_{n}$ and $p q=\sum_{i=1}^{n} \mathbf{p}_{i} \cdot \mathbf{q}_{i}$. It is a well-known fact that, in the obvious notation, $\phi(q)$ is the inverse Fourier 
transform of $\hat{\phi}(p)$ :

$$
\phi(q)=F \hat{\phi}=(2 \pi)^{-3 n / 2} \int e^{-i p q} \hat{\phi}(p) d p .
$$

In the $q$-representation, the $j$ th position operator $\mathbf{q}_{j}$ is represented by multiplication by $\mathbf{q}_{j}$, while the $j$ th momentum operator $\mathbf{p}_{j}$ is represented by $(1 / i)\left(d / d \mathbf{q}_{j}\right)$ on their natural domains. The $p$-representation has the momentum $\mathbf{p}_{j}$ represented by multiplication by $\mathbf{p}_{j}$ and the position $\mathbf{q}_{j}$ represented by $-(1 / i)\left(d / d \mathbf{p}_{j}\right)$. If $\phi$ is a vector in $L^{2}(\mathbf{R})$ the ray to which it belongs will be denoted by $\phi$, and keeping in mind the fact that vectors are equivalence classes of functions in $L^{2}\left(\mathbf{R}^{3 n}\right)$ we will usually write a vector $\phi$ as a function $\phi(q)$. Also it is profitable for our work to denote a state of the system as a pair $[\phi(q), \hat{\phi}(p)]$ even though this notation involves a redundancy.

In this notation the Pauli problem is the following: Does an exact determination of both the probability distribution $|\phi(q)|^{2}=|\phi(q)|^{2}$ in the position coordinates and the probability distribution $|\hat{\phi}(p)|^{2}=|\hat{\phi}(p)|^{2}$ in the momentum coordinate define the state $[\phi(q), \hat{\phi}(p)]$ unequivocally? Notice that the Pauli data involves a pair of functions $[|\Phi(q)|,|\hat{\phi}(p)|]$ from which we wish to determine the ray $[\phi(q), \hat{\phi}(p)]$. There is no loss of generality in determining a function $[\phi(q), \hat{\phi}(p)]$ in the equivalence class defined by the vector, and we will always look for a functional representative of the state that is as smooth as possible. Also the term wave function will be used to denote a Lebesgue square integrable function.

In this sense the Pauli data can be thought of as the pair of functions $[|\phi(q)|,|\hat{\phi}(p)|]$. However, there is another way of presenting the Pauli data that is sometimes useful. Let $u(q)$ and $w(p)$ be any pair of complex-valued functions of $q$ and $p$ respectively. $u(q)$ and $w(p)$ can then be considered as multiplicative operators in the $q$ - and $p$-representations respectively. Let us denote by $E_{\chi}(A)$ the expectation value of observable $A$ in the state $\chi$,

$$
E_{\chi}(A)=(\chi, A \chi)
$$

Then we have the following result:

Proposition 1. The state $[\psi, \hat{\psi}]$ satisfies the Pauli data $[|\phi(q)|,|\hat{\phi}(p)|]$ if and only if both

$$
E_{\psi}(u)=\int u(q)|\phi(q)|^{2} d q
$$

and

$$
E_{\psi}(w)=\int w(p)|\hat{\phi}(p)|^{2} d p
$$

for all functions $u$ and $w$. The equations (4) and (5) are to be understood in the extended sense that if the right side is undefined then so is the left side. 
Proof. The only if direction is obvious. The argument in the if direction follows by taking $u(q)$ to be the characteristic function of an arbitrary Borel set of $\mathbf{R}$. Then the measures $|\phi(q)|^{2} d q$ and $|\psi(q)|^{2} d q$ assign the same measure to any Borel set. Hence by possibly adjusting the values of $\psi(q)$ on a set of measure zero, we get $|\psi(q)|=|\phi(q)|$. The same argument works for $|\hat{\psi}(p)|=|\hat{\phi}(p)|$.

The result means that any state satisfying a given set of Pauli data must have the same expectation values for any operators of the form $\alpha u(q)+\beta w(p)+\delta I, \alpha, \beta$ and $\delta$ are complex numbers, $u(q)$ and $w(p)$ are operator-valued functions of the seifadjoint operators $q$ and $p$.

This leads to the following characterization of non unique Pauli states.

Proposition 2. The state $[\phi, \hat{\phi}]$ is Pauli non-unique if and only if there exists a pair of non-trivial self-adjoint operators $F$ and $G$, with

and

$$
(F \phi)(q)=f(q) \phi(q), \quad \forall \phi \in D(F)
$$

such that

or equivalently

$$
(G \phi)^{\wedge}(p)=g(p) \hat{\phi}(p), \quad \forall \phi \in D(G)
$$

REMARKS. Equation (8) can be written in terms of rays

that is

$$
\left(e^{i F} e^{i q} \phi\right)(q)=\phi(q),
$$

$$
\left(e^{i F} e^{i \alpha} \phi\right)^{\wedge}(p)=\hat{\phi}(p) .
$$

$$
\left(e^{i F} e^{i q} \phi\right)(q)=\phi(q),
$$

$$
\left(e^{i F} e^{i \theta} e^{i \lambda} \phi\right)(q)=e^{i \mu} \phi(q)
$$

but by absorbing the real constants $\lambda$ and $\mu$ into either $F$ or $G$ we observe that we have suffered no loss of generality in using vectors instead of rays.

The adjective non-trivial removes the possibility that both $F$ and $G$ are merely constant multiples of the identity operator.

\section{ProOF}

Sufficiency: If $\psi=e^{-i F} \phi=e^{i G} \phi$ then the expression of the Pauli data in equations (4) and (5) can be used to show that $[\psi, \hat{\psi}]$ and $[\phi, \hat{\phi}]$ satisfy the same Pauli data.

Necessity: If $\psi$ and $\phi$ satisfy the same Pauli data then the pair $[|\psi(q)|,|\psi(p)|]$ equals the pair $[|\phi(q)|,|\hat{\phi}(p)|]$. Therefore there exist real functions $f$ and $g$ such that

and

$$
\psi(q)=e^{-i f(q)} \phi(q)
$$

$$
\hat{\psi}(p)=e^{i g(p)} \hat{\phi}(p)
$$


but

$$
\begin{aligned}
\psi(q) & =F \hat{\psi}(p) \\
& =F e^{i g(p)} F F \phi(p) \\
& =\left(e^{i g(p)} \phi\right)(q),
\end{aligned}
$$

where in the last expression $p=(1 / i)(d / d q)$. Thus $e^{-i f(q)} \phi(q)=\left(e^{i g(p)} \phi\right)(q)$, or, in abstract notation,

$$
e^{-i F} \phi(q)=e^{i G} \phi(q),
$$

where $F$ and $G$ are self-adjoint operators which are multiplicative in the $q$ - and $p$-representations respectively. This proposition has two immediate corollaries that we will use in the construction of examples of non-unique Pauli states.

COROLlARY 1. If $A$ is a self-adjoint operator such that $e^{i A}=e^{i F} e^{i G}$, where $F$ is a self-adjoint operator function of $q$ and $G$ is a self-adjoint operator function of $p$, then any eigenfunction of $A$ is a Pauli non-unique state.

COROLlaRY 2. Let A be a self-adjoint operator such that $e^{i A}$ is unitarily equivalent to the unitary operator $e^{i F} e^{i G}$, with $F$ and $G$ given in Corollary 1 , that is

$$
U e^{i A} U^{*}=e^{i F} e^{i G}
$$

for some unitary operator $U$. If $\phi_{\lambda}$ is an eigenfunction of $A, A \phi_{\lambda}=\lambda \phi_{\lambda}$, then $\psi_{\lambda}=U \phi_{\lambda}$ is a Pauli non-unique state, $\left[\psi_{\lambda}, \hat{\psi}_{\lambda}\right]$.

The central problem in constructing Pauli non-unique states is to sum the Campbell-Baker-Hausdorff formula for $e^{i F} e^{i G}$. In the next section we will give one example that uses the structure of the non-compact Lie group $S L(2, \mathbf{R})$ to sum this formula and one example that does not depend upon this formula.

The constructions developed in the next two sections will be restricted to the case of one degree of freedom, namely $\mathscr{H}=L^{2}(\mathbf{R})$. We will take up the general case again in Section 5.

One further consequence of our notation should be noted. We say that a state $[\phi(q), \hat{\phi}(p)]$ is a real state or real-valued state, if there exists a representative of $[\phi(q), \hat{\phi}(p)]$ as a vector pair $[\phi(q), \hat{\phi}(p)]$ in which either $\phi(q)$ or $\hat{\phi}(p)$, or both, are real-valued functions. If no such representative exists then the state is strictly complex-valued or non-real valued. An analogous statement holds for parity and the complex representative of that state in which $\hat{\phi}(-q)= \pm \hat{\phi}(q)$ (and hence $\hat{\phi}(-p)= \pm \hat{\phi}(p))$.

In fact, whenever we assert that a state $[\phi(q), \hat{\phi}(p)]$ has a certain functional property we always mean that there is a representative of it that has this property. 


\section{The construction of Pauli non-unique states}

This section is concerned with the construction of examples of states that are Pauli non-unique. We restrict ourselves to the Hilbert space $L^{2}(\mathbf{R})$; the extension of these constructions to $L^{2}\left(\mathbf{R}^{n}\right), n>1$, is immediate but will not be discussed here.

We present two sets of examples of non-unique states; these sets are not disjoint. The first set contains states of definite parity that are not real-valued. For these states the complex conjugate state is the Pauli partner state.

In the second set of examples we construct a two-parameter family of subsets of Pauli non-unique states that are parameterized in the following way. Let $H(m, K)=\left(p^{2} / 2 m\right)+\left(K q^{2} / 2\right)$ be the Hamiltonian for the one-dimensional harmonic oscillator with mass $m$ and constant $K$, that are both positive real numbers. We define constants $\beta=(K m)^{\frac{1}{2}}$ and $\alpha=(K / m)^{\frac{1}{2}}$ and assume that $\alpha=2 \pi / b$ for some integer $b \geqslant 3$. For any such integer $b \geqslant 3$ and integer $c, 0 \leqslant c \leqslant b-1$, let $\mathscr{H}_{b, c}$ be the subspace of $L^{2}(\mathbf{R})$ spanned by the states $\left\{\psi_{n b+c}\right\}_{n=0}^{\infty}$, where

$$
\psi_{n b+c}(q)=e^{i s q^{2} / 4} \phi_{n b+c}(q)
$$

In this expression, $s=2 \beta(1-\cos \alpha) / \sin \alpha$ and $\phi_{n b+c}(q)$ is the $(n b+c)$ th harmonic oscillator eigenfunction for the harmonic oscillator Hamiltonian $H(m, K)$.

The closed subspaces $\mathscr{H}_{b, c}$ will be shown to be subspaces of Pauli non-unique states if $b \geqslant 3$.

For fixed $b \geqslant 3$, that is a fixed ratio $K / m$, there is a one parameter family of harmonic oscillator Hamiltonians $H(\mathrm{~m}, K)$ that can be used to develop the $\mathscr{H}_{b, c}$. Different choices of the Hamiltonian in this family give different values to a parameter $\gamma$ in the eigenfunctions $\left\{\phi_{n}(q)\right\}$. With $\alpha, \beta$ fixed and $\gamma=(\beta / 2)^{\frac{1}{2}}$, the nth eigenfunction of $H(K, m)$ satisfying

$$
H(K, m) \phi_{n}=\left(n+\frac{1}{2}\right) \alpha \phi_{n}, \quad n=0,1,2, \ldots
$$

is

$$
\phi_{n}(q)=\frac{(-1)^{n}}{2^{n} \gamma^{n-1} \pi^{\frac{1}{2}}(n !)^{\frac{1}{2}}} \exp \left(\gamma^{2} q^{2}\right)\left(\frac{d}{d q}\right)^{n} \exp \left(-2 \gamma^{2} q^{2}\right)
$$

Furthermore, for each fixed pair $m, K$ with

$$
\frac{K}{m}=\frac{(2 \pi)^{2}}{b^{2}} \leqslant \frac{(2 \pi)^{2}}{9}
$$

the set theroretic union of the subspaces $\left\{\mathscr{H}_{b, c}\right\}, c=0,1, \ldots, b-1$, is a fundamental subset of $L^{2}(\mathbf{R})$. Hence we have a two-parameter family of fundamental subsets of Pauli non-unique states.

On the other hand, although every state in $L^{2}(\mathbf{R})$ can be approximated arbitrarily well by Pauli non-unique states, not every state is Pauli non-unique. For example, 
any eigenfunction $\phi_{n}(q)$ can be obtained as the limit of a sum of non-unique states, but this method gives $\phi_{n}(q)$ as its own Pauli partner.

We will now present these two sets of examples in more detail.

EXAMPle 1. Any state of definite parity satisfies Pauli data that is also satisfied by its complex conjugate. Therefore any state of definite parity that is not realvalued is a Pauli non-unique state.

Let $J$ be the conjugation operator; $J: L^{2}(\mathbf{R}) \rightarrow L^{2}(\mathbf{R}),(J \phi)(q)=\overline{\phi(q)}$ for all $\phi(q) \in L^{2}(\mathbf{R})$, and let $F$ denote, as before, the Fourier transform map. Then $\phi(q)$ and $(J \phi)(q)$ satisfy the same Pauli data if

$$
(F J \phi)(p)= \pm(J F \phi)(p) .
$$

This equation is satisfied if $\phi(-q)= \pm \phi(q)$. Therefore if $\phi(q)$ has definite parity and $\phi(q)$ is not real-valued, then the states $[\phi(q), \hat{\phi}(p)]$ and $[\bar{\phi}(q), \hat{\bar{\phi}}(p)]$ are distinct states that satisfy the same Pauli data and are Pauli partner states.

EXAMPLE 2. In our earlier discussion of necessary conditions for the existence of Pauli non-unique states, we observed, in Corollary 2 , that if a state $\phi \in L^{2}(\mathbf{R})$ satisfies an eigenvalue equation $A(p, q) \phi=\lambda \phi$ for some self-adjoint operator $A$ and if there exists a unitary operator $U$ such that

$$
U e^{i A} U^{-1}=e^{i F} e^{i G},
$$

where $F=F(q), G=G(p)$ are self-adjoint operators, then $U \phi$ is Pauli non-unique. We will use $U=e^{i F / 2}$ to obtain a class of non-unique states in this way.

For our purpose the self-adjoint operator $A$ is the Hamiltonian for the harmonic oscillator,

$$
H(m, K)=\frac{p^{2}}{2 m}+\frac{K q^{2}}{2} .
$$

There is a Hamiltonian $H(m, K)$ for each pair of positive real numbers $(m, K)$; we will also need the real numbers $\alpha=(K / m)^{\ddagger}$ and $\beta=(K m)^{\sharp}$. In Appendix 1 we prove that if $\alpha=(K / m)^{\sharp} \neq n \pi$ for any integer $n$ then

$$
\exp \left(i s q^{2} / 4\right) \exp i H(m, K) \exp \left(-i s q^{2} / 4\right)=\exp \left(i s q^{2} / 2\right) \exp \left(-i t p^{2} / 2\right)
$$

with the real numbers $s$ and $t$ given by

$$
\left.\begin{array}{l}
s=2 \beta(1-\cos \alpha) / \sin \alpha, \\
t=\sin \alpha / \alpha .
\end{array}\right\}
$$

Therefore, taking $U=\exp \left(i s q^{2} / 4\right)$ in the above equation, we have that the wave functions

$$
\psi_{n}(q)=\exp \left(i s q^{2} / 4\right) \phi_{n}(q), \quad n=0,1,2, \ldots
$$


are representatives of Pauli non-unique states, because

$$
\exp \left(i s q^{2} / 2\right) \exp \left(-i t p^{2} / 2\right) \psi_{n}(q)=\exp \left(i \lambda_{n}\right) \psi_{n}(q)
$$

where $\lambda_{n}$ is the $n$th eigenvalue of the operator $H(m, K), \lambda_{n}=\left(n+\frac{1}{2}\right) \alpha$. In fact the Pauli partner state of $\psi_{n}$ has a representative

$$
\psi_{n}^{\prime}(q)=\exp \left(-i s q^{2} / 2\right) \psi_{n}(q)=\exp \left(-i s q^{2} / 4\right) \phi_{n}(q)=\overline{\psi_{n}(q)}
$$

Since the $\left\{\phi_{n}\right\}_{n=0}^{\infty}$ are real states and have definite parity, this construction appears to achieve nothing more than is obtained in Example 1, except that we have explicitly displayed a two-parameter family of complete orthonormal bases for $L^{2}(\mathbf{R})$ in which each member of each basis in the family is Pauli non-unique.

However, this construction can be extended to give $L^{2}(\mathbf{R})$ as a direct sum of subspaces of Pauli non-unique states in a countable infinity of ways. The nonunique states in these subspaces are not necessarily of definite parity and do not, in general, take their complex conjugate as a Pauli partner.

For any integer $b \geqslant 3$, and integer $c, 0 \leqslant c \leqslant b-1$, let $\mathscr{H}_{b, c}$ be the subspace of $L^{2}(\mathbf{R})$ spanned by the states $\left\{\psi_{n b+c}\right\}_{n=0}^{\circ}$. Again we use the notation of equation (17),

$$
\psi_{n b+c}(q)=\exp \left(i s q^{2} / 4\right) \phi_{n b+c}(q),
$$

where the harmonic oscillator Hamiltonian $H(K, m)$ has coefficients $m$ and $K$ such that $\alpha=(K / m)^{\frac{t}{2}}=2 \pi / b$. Then

$$
s=4 \pi m(1-\cos (2 \pi / b)) / b \sin (2 \pi / b) \text { and } t=b \sin (2 \pi / b) / 2 \pi
$$

are finite for integers $b \geqslant 3$. For fixed values of $m$ and $K$ satisfying $\alpha=2 \pi / b$, the subspaces $\mathscr{H}_{b, c}$ and $\mathscr{H}_{b, c^{\prime}}$ are orthogonal if $c \neq c^{\prime}$, because $\psi_{k}$ is orthogonal to $\psi_{l}$ if $k \neq l$ : Furthermore, since the $\left\{\psi_{k}\right\}_{k=0}^{\infty}$ form a basis for $L^{2}(\mathbf{R})$ we have the direct sum decomposition,

$$
L^{2}(\mathbf{R})=\underset{c=0}{\oplus-1} \mathscr{H}_{b, c}
$$

THEOREM 1. The subspaces $\mathscr{H}_{b, c}$ defined above are subspaces of $L^{2}(\mathbf{R})$ all of whose members are Pauli non-unique states provided that $b \geqslant 3$.

Proor. We will show that any state $\xi \in \mathscr{H}_{b, c}$ satisfies

$$
\exp \left(i s q^{2} / 2\right) \exp \left(-i t p^{2} / 2\right) \xi=\exp ((2 c+1) \pi / b) \xi
$$

where $s$ and $t$ are given in equation (19) for $b \geqslant 3$. Then Corollary 2 of Section 2 proves that each $\xi$ is Pauli non-unique. 
Any $\xi(q) \in \mathscr{H}_{b, c}$ is of the form

$$
\xi(q)=\lim _{N \rightarrow \infty} \xi_{N}(q),
$$

where $\xi_{N}(q)=\sum_{n=0}^{N} a_{n} \psi_{n b+c}(q)$ and the complex number $a_{n}=\left(\xi, \psi_{n b+c}\right)$. But by construction

$$
\exp \left(i s q^{2} / 2\right) \exp \left(-i t p^{2} / 2\right) \xi_{N}(q)=\exp (i(2 c+1) \pi / b) \xi_{N}(q)
$$

because the $(n b+c)$ th eigenvalue of $H(K, m)$ with $\alpha=2 \pi / b$ is

$$
\lambda_{n b+c}=\left(n b+c+\frac{1}{2}\right) 2 \pi / b=n 2 \pi+(2 c+1) \pi / b
$$

and hence $\exp \left(i \lambda_{n b+c}\right)$ is independent of $n$.

However, $\exp \left(i s q^{2} / 2\right) \exp \left(-i t p^{2} / 2\right)$ is a unitary operator and hence by a standard argument we can take the limit and get the desired result.

REMARKS. It should be noted that when we change $b$, that is, we pass from one family of subspaces $\left\{\mathscr{H}_{b, c}\right\}_{c=0}^{b-1}$ to another with different integer $b \geqslant 3$, we change the parameters $m, K$ of the harmonic oscillator Hamiltonian $H(K, m)$. Nevertheless, for a fixed $b$, there is a one-parameter family of harmonic oscillator Hamiltonians, namely, those with $(K / m)^{\mathbf{t}}=2 \pi / b$, that can be used to develop the $\mathscr{H}_{b, c}$. Different choices of the Hamiltonian in this family will give different parameters $\gamma$ in the harmonic oscillator eigenfunctions (13) and therefore for fixed $b$ we have a oneparameter, $\gamma$ or $\beta$, family of different subspaces $\mathscr{H}_{b, c}$ of non-unique states.

Furthermore, for each fixed pair $m, K$ with $\alpha=2 \pi / b$ and $\beta$ fixed, we have a fundamental subset of Pauli non-unique states, namely the set theoretical union of the subspaces $\mathscr{H}_{b, c}, c=0, \ldots, b-1$. Therefore we have constructed a twoparameter family of fundamental subsets, $D(\alpha=2 \pi / b, \beta)$, of Pauli non-unique states.

\section{The construction of Pauli unique states}

In the previous section all the Pauli non-unique states that we found were nonreal, so it is not surprising that our main results here involve real states. We have not been able to prove that all real states are Pauli unique, but have found a large class of real states that are unique. The limits of this class of unique states are prescribed more by our methods of proof than by any natural conditions.

We begin by proving that a large class of real states are Pauli unique. This class contains the bound states of time-reversal invariant Hamiltonian operators $p^{2} / 2 m+V(q)$. A different method is then used to obtain the result that any complex 
Gaussian wave function

$$
\phi_{\alpha \beta \gamma}(q)=\frac{1}{(2 \pi \gamma)^{\frac{1}{t}}} \exp \left\{-\frac{(q-\beta I)^{2}}{4 \gamma}+i \alpha q\right\}
$$

is Pauli unique.

In order to obtain examples of Pauli unique states we formulate the Pauli data in terms of expectation values of arbitrary functions of $q$ and of arbitrary functions of $p$, that we call bits of Pauli data. Given a set of Pauli data $[|\phi(q)|,|\hat{\phi}(n)|]$ in the form of a set of extended complex numbers $\{E \phi(f), E \phi(g)\}$ for arbitrary functions $f(q)$ and $g(p)$, it turns out to be useful to examine the relations between the various bits. If a subset of the bits obeys relations amongst its members that can only be satisfied by a single ray of vectors then that Pauli data uniquely defines a state.

We will construct Pauli unique states using only a finite number of bits of Pauli data. What we have in mind are collections of equations and inequalities of the form

or

$$
\left.\begin{array}{l}
E \phi(f) E \phi(g) \geqslant E \phi(h), \\
E \phi(1)=1, \\
E \phi(f)=\alpha, \quad E \phi(h)=\beta
\end{array}\right\}
$$

$$
\left.\begin{array}{l}
E \phi(f)+E \phi(g) \geqslant E \phi(h), \\
E \phi(1)=1, \\
E \phi(f)=\alpha, \quad E \phi(h)=\beta,
\end{array}\right\}
$$

where $f, g$ and $h$ are given functions of either $p$ or $q$ alone or a sum of such functions and $\alpha$ and $\beta$ are fixed complex numbers. In general the inequalities are assumed to hold for some class of states $\phi$. Examples of (2I) include the uncertainty relations and examples of (22) include the semi-boundedness of the energy. If it is known that the inequalities in (21) become equalities for a single ray fixed by the other equations, then the Pauli data that satisfies all the equations in (21) determines that state uniquely. On the other hand, if it is known that the inequality in (22) is satisfied by a particular state then it may be possible to show that the Pauli data uniquely determines that state.

A typical system in the form of (22) is associated with operators $A$ that are semi-bounded and of the form

$$
A=f(p)+g(q)
$$

with $f$ and $g$ real measurable functions and $h$ is the zero operator. In particular if we take $f(p)=p^{2}, g(q)=0$ or $f(p)=0, g(q)=q^{2}$ we get a large class of real states that are Pauli unique. 
THEOREM 2. Let $\{\phi(q), \hat{\phi}(p)\}$ be a real state, then it is a Pauli unique if, either

(a) it has a real representative $\phi(q)$ and $E_{\phi}\left(p^{2}\right)<\infty$

or (b) it has a real representative $\hat{\phi}(p)$ and $E_{\phi}\left(q^{2}\right)<\infty$.

Proof. We will assume that the state $\{\phi(q), \hat{\phi}(p)\}$ has a real representative $\phi(q)$ and $E_{\phi}\left(p^{2}\right)<\infty$, that is case (a) holds, the other case can be handled in a like manner.

Let $\{\Psi(q), \hat{\Psi}(p)\}$ be a Pauli partner of the given state. It follows that $|\phi(q)|=|\psi(q)|$ almost everywhere in $\mathbf{R}$, for any representative $\psi(q)$ of $\{\psi(q), \hat{\Psi}(p)\}$. Therefore $\psi(q)=e^{i g(q)} \phi(q)$ almost everywhere in $\mathbf{R}$ where $g(q)$ is a real-valued function. On the other hand, $\left(\phi, p^{2} \phi\right)=\left(\psi, p^{2} \psi\right)<\infty$. A direct calculation of the real part of this equation yields $\left(\phi,\left(g^{\prime}\right)^{2} \phi\right)=0$, hence $g(q)$ is constant almost everywhere and $\psi(q)$ is a representative of $\{\phi(q), \hat{\phi}(p)\}$.

In physical terms this result says that for a spinless one-dimensional particle every motion reversal invariant state with a finite mean kinetic energy is Pauli unique. Mathematically, any real function in the domain of the self-adjoint differential operator $p=(1 / i)(d / d q)$ is a Pauli unique.

This class of Pauli unique real states can be enlarged to include states that are not in the domain of $p$ by the following method.

THEOREM 3. Let $A=p^{2}+w(q)$ be a real operator. Then any real state $\{\phi(q), \hat{\phi}(p)\}$ with real representative $\phi(q)$ is Pauli unique if $E_{\phi}(A)<\infty$.

ReMARK. A similar theorem holds if $A$ is of the form $A=q^{2}+u(p)$, and the state has real representative $\hat{\phi}(p)$ with $E_{\phi}(A)<\infty$.

Proof. The proof follows the lines of the previous theorem, the main difference is that $E_{\phi}\left(p^{2}\right)$ need not be finite.

As before, let $\{\phi(q), \hat{\phi}(p)\}$ be a Pauli partner of the given state $\{\phi(q), \hat{\phi}(p)\}$. Now we must have $(\phi, A \phi)=(\psi, A \psi)$, for any representative $\psi(q)$ of $(\psi(q), \hat{\psi}(p)\}$. A direct calculation yields, for $\psi(q)=e^{i g(q)} \phi(q)$ a.e., that $g^{\prime}(q)=0$ a.e. and hence $\psi(q)$ is a representative of $\{\phi(q), \hat{\phi}(p)\}$.

While this theorem does extend the class of real states that are Pauli unique, for example any continuous function with a piecewise continuous first derivative is Pauli unique, it is not strong enough to encompass all real states. Its most important consequence is that it shows that bound states in quantum mechanics are Pauli unique.

COROLlARY. If $H=p^{2} / 2 m+V(q)$ is a motion-reversal invariant (real) Hamiltonian operator, then every bound state of $H$ is Pauli unique. 
Proof. We have only to note that any bound state of $H$ has a real representative $\phi(q)$ and then use Theorem 3.

Systems of the form of equation (21) are typically of a form associated with the Heisenberg uncertainty relations. Recall that for all $\phi \in D(p) \cap D(q)$, the commutation relation $i[p, q] \subset I$, implies that the uncertainty relations $\|p \phi\|^{2}\|q \phi\|^{2} \geqslant \frac{1}{4}\|\phi\|^{2}$ hold. Equality is attained in this expression for the unique normalized state $\phi(q)=(2 \pi)^{-1} e^{-q^{2} / 2}$ which satisfies $(p-i q) \phi=0$. The proof of this well-known fact is given, for example, in [5] and [1]. We will need a slight generalization of this which we will now prove.

Proposition 3. Let $A, B$ and $C$ be real linear operators with non-trivial domains $D(A), D(B)$ and $D(C)$, subsets of a separable Hilbert space $\mathscr{H}$ and which are not necessarily dense in $\mathscr{H}$. Let $\Omega$ be a non-trivial subset of $D(A) \cap D(B)$ such that for all $\phi \in \Omega$ we have

then for all $\phi \in \Omega$,

$$
(A \phi, B \phi)-(B \phi, A \phi)=i(\phi, C \phi)
$$

$$
\|A \phi\|^{2}\|B \phi\|^{2} \geqslant \frac{1}{1}(\phi, C \phi)^{2} .
$$

Furthermore, $\|A \phi\|^{2}\|B \phi\|^{2}=\frac{1}{4}(\phi, C \phi)^{2}<\infty$ with $\|B \phi\| \neq 0$, if and only if $\phi$ satisfies

where

$$
A \phi+\lambda B \phi=0
$$

$$
\lambda=-i(\phi, C \phi) / 2\|B \phi\|^{2} .
$$

Proof. We observe that $(\phi, C \phi)=2 \operatorname{lm}(B \phi, A \phi)$ for all $\phi \in \Omega$, and therefore

$$
\begin{aligned}
\frac{1}{2}|(\phi, C \phi)| & \leqslant|(B \phi, A \phi)| \\
& \leqslant\|B \phi\|\|A \phi\| \quad \text { by Schwarz' inequality. }
\end{aligned}
$$

It follows from Schwarz' inequality that equality is obtained only for those vectors $\phi$ that satisfy $A \phi+\lambda B \phi=0$ for some complex number $\lambda$. Moreover, equality is obtained in the first step if and only if $\operatorname{Re}(B \phi, A \phi)=0$. Therefore for equality to hold we require $\operatorname{Re} \lambda\|B \phi\|^{2}=0$, and if $B \phi \neq 0$, this means that $\operatorname{Re} \lambda=0$. Putting this together we get that $\|A \phi\|\|B \phi\|=\frac{1}{2}|(\phi, C \phi)|$, with $\|B \phi\| \neq 0$ if and only if $A \phi=-\lambda B \phi$, where $\lambda=-i(\phi, C \phi) / 2\|B \phi\|^{2}$.

This result will be useful when $A$ and $B$ are real operator-valued functions of $p$ or of $q$, and $C$ is an operator-valued function of $p$ and $q$ of the form $f(q)+g(p)$. For example, if we take $A=p-\alpha I, B=q-\beta I$, where $\alpha$ and $\beta$ are real numbers, and $C=I$ the identity operator, then for all $\phi \in D(p) \cap D(q)$ we have that

$$
\|(p-\alpha I) \phi\|^{2}\|(q-\beta I) \phi\|^{2} \geqslant \frac{1}{4}\|\phi\|^{2} .
$$


This becomes an equality for states $\psi$ that satisfy

$$
(p-\alpha I) \psi=\frac{i(q-\beta I) \psi}{2 \gamma},
$$

where $\gamma=\|(q-\beta I) \psi\|^{2}$. If $\psi$ is assumed to be normalized, $\|\psi\|=1$, for fixed $\alpha$ and $\beta$ we get a one-parameter family of states $\left\{\phi_{\gamma}\right\}$ that satisfy equation (27).

This argument leads to a class of Pauli unique states, the set of states $\left\{\phi_{\alpha \beta \gamma}\right\}$ parameterized by the three real numbers $\alpha, \beta, \gamma$, where

and

$$
\left.\begin{array}{l}
\left\|(q-\beta I) \phi_{\alpha \beta \gamma}\right\|^{2}=\gamma,\left\|\phi_{\alpha \beta \gamma}\right\|=1 \\
\left\|(p-\alpha) \phi_{x \beta \gamma}\right\|^{2}=1 / 4 \gamma .
\end{array}\right\}
$$

For given $\alpha, \beta, \gamma, \phi_{\alpha \beta \gamma}$ has a representative

$$
\phi_{\alpha \beta \gamma}(q)=(2 \pi \gamma)^{-1} \exp \left(-(q-\beta I)^{2} / 4 \gamma+i \alpha q\right) .
$$

These states are Pauli unique because the equations (3) can be presented as expectations. $E \phi_{\alpha \beta \gamma}\left((q-\beta I)^{2}\right)=\gamma$, for example, that make up part of the Pauli data and that can only be satisfied by states that are solutions of equation (27). In fact the function $\phi_{\alpha \beta \gamma}(q)$ has an even real part and an odd imaginary part and therefore $\phi_{\alpha \beta \gamma}(q)$ is the inverse Fourier transform of a real function $\hat{\phi}_{\alpha \beta \gamma}(p)$. Therefore the states $\left\{\phi_{\alpha \beta \gamma}(q), \hat{\boldsymbol{\phi}}_{\alpha \beta \gamma}(p)\right.$ are examples of real states that are Pauli unique through the arguments of Theorem 2 because they all belong to the domain of $q^{2}$.

There are still two questions to be answered. Are all real states Pauli unique? Are the real states the only states that are Pauli unique? The above work points to an answer of yes for both these questions, but they do not seem capable of being answered within the framework we have used.

\section{Extension to $L^{2}\left(\mathbf{R}^{n}\right), n>1$}

The extension of the results of the two previous sections to Hilbert spaces $L^{2}\left(\mathbf{R}^{n}\right)$ for $n>1$ is achieved by writing $\left.L^{2} \mathbf{R}^{n}\right)$ as a tensor product of the spaces $L^{2}(\mathbf{R})$,

$$
L^{2}\left(\mathbf{R}^{n}\right)=\bigotimes_{j=1}^{n} \mathscr{H}_{j}
$$

where $\mathscr{H}_{j}=L^{2}(\mathrm{R})$ for each $j=1, \ldots, n$. We shall use the notation that a vector $\Phi\left(q_{1}, \ldots, q_{n}\right)=\phi_{1}\left(q_{1}\right) \times \phi_{2}\left(q_{2}\right) \times \ldots \times \phi_{n}\left(q_{n}\right)$ that belongs to $L^{2}\left(\mathbf{R}^{n}\right)$ will be written as

$$
\Phi\left(q_{1}, \ldots, q_{n}\right)=\phi_{1}\left(q_{1}\right) \phi_{2}\left(q_{2}\right) \ldots \phi_{n}\left(q_{n}\right) .
$$

We will discuss the various examples of uniqueness and non-uniqueness in turn. 
Pauli non-unique states in $L^{2}\left(\mathbf{R}^{n}\right)$

It is easier to construct non-unique states in $L^{2}\left(\mathbf{R}^{n}\right)$ because if $\phi_{j}\left(q_{j}\right)$ is nonunique in $L^{2}(\mathbf{R})$ with Pauli partner $\psi_{j}\left(q_{j}\right)$ then the state $\phi_{1}\left(q_{1}\right) \phi_{2}\left(q_{2}\right) \ldots \phi_{j}\left(q_{j}\right) \ldots \phi_{n}\left(q_{n}\right)$ is non-unique in $L^{2}\left(\mathbf{R}^{n}\right)$ with Pauli partner $\phi_{1}\left(q_{1}\right) \phi_{2}\left(q_{2}\right) \ldots \psi_{j}\left(q_{j}\right) \ldots \phi_{n}\left(q_{n}\right)$. This is true no matter what choice is made for $\phi_{i}\left(q_{i}\right), i \neq j$. We now list the specific examples.

(i) If $\Phi\left(q_{1}, \ldots, q_{n}\right)$ is strictly complex-valued and $\Phi\left(-q_{1}, \ldots,-q_{n}\right)= \pm \Phi\left(q_{1}, \ldots, q_{n}\right)$ then $\left[\Phi\left(q_{1}, \ldots, q_{n}\right), \Phi\left(p_{1}, \ldots, p_{n}\right)\right]$ and $\left[\bar{\Phi}\left(q_{1}, \ldots, g_{n}\right), \hat{\Phi}\left(p_{1}, \ldots, p_{n}\right)\right]$ are distinct states that satisfy the same Pauli data.

(ii) Let $b=\left(b_{1}, \ldots, b_{n}\right)$ and $c=\left(c_{1}, \ldots, c_{n}\right)$ be multi-indices, and define for $b_{j} \geqslant 3,0 \leqslant c_{j} \leqslant b_{j}-1, j=1, \ldots, n, \mathscr{H}_{b c}=\otimes_{j=1}^{n} \mathscr{H}_{b_{i}, c}$, where

$$
\mathscr{H}_{b_{n} c_{j}}=\left\{\psi_{n b+c}\left(q_{g}\right)\right\}_{n=0}^{\infty} \subset L^{2}\left(\mathbf{R}^{n}\right) .
$$

Here $\psi_{n b_{j}+c_{j}}\left(q_{j}\right)=\exp \left(i s_{j} q_{j}^{2} / 4\right) \phi_{n b_{j}+c_{j}}\left(q_{j}\right), \phi_{n b_{j}+c_{1}}\left(q_{j}\right)$ is the $\left(n b_{j}+c_{j}\right)$ th eigenfunction of the harmonic oscillator Hamiltonian $H\left(m_{j}, K_{j}\right)$ with

$$
\alpha_{j}=\left(K_{j} / m_{j}\right)^{\frac{1}{2}}=2 \pi / b_{j},
$$

and $s_{j}=2 \beta_{j}\left(1-\cos \alpha_{j}\right) / \sin \alpha_{j}$, where $\beta_{j}=\left(m_{j} K_{j}\right)^{\frac{1}{1}}$. By the argument of Proposition 3 the subspaces $\mathscr{H}_{b, c}$ with $b_{j} \geqslant 3$ for each $j=1, \ldots, n$, are subspaces all of whose members are Pauli non-unique. It also follows that we have thereby constructed a $2 n$ parameter family of fundamental subsets of Pauli non-unique states.

\section{Pauli unique states}

In so far that it is easier to construct non-unique states it is more difficult to construct unique states in $L^{2}\left(\mathbf{R}^{n}\right)$. However, the construction in $L^{2}(\mathbf{R})$ of Pauli unique states has an immediate generalization to $L^{2}\left(\mathbf{R}^{n}\right)$. Moreover, this generalization does not depend upon the tensor product decomposition of $L^{2}\left(\mathbf{R}^{n}\right)$.

Theorem 6. Let $\left\{\phi\left(q_{1}, \ldots, q_{n}\right), \hat{\phi}\left(p_{1}, \ldots, p_{n}\right)\right\}$ be a real state, then it is Pauli unique if, either

(a) it has a real representative $\phi\left(q_{1}, \ldots, q_{n}\right)$ and $E_{\phi}\left(p^{2}\right)<\infty$

or $(b)$ it has a real representative $\hat{\phi}\left(p_{1}, \ldots, p_{n}\right)$ and $E_{\phi}\left(q^{2}\right)<\infty$ where $p^{2}=\sum_{i=1}^{n} p_{i}^{2}$ and $q^{2}=\sum_{i=1}^{n} q_{i}^{?}$.

Proof. The proof follows exactly the same line as the proof of Theorem 2. A direct calculation of $\left(\psi, p^{2} \psi\right)=\left(\phi, p^{2} \phi\right)$ yields, for

$$
\left.\psi\left(q_{1}, \ldots, q_{n}\right)=\exp \left\{i g q_{1}, \ldots, q_{n}\right)\right\} \phi\left(q_{1}, \ldots, q_{n}\right), \quad \sum_{i=1}^{n}\left(\frac{\partial g}{\partial q_{i}}\right)^{2}=0 \text { a.e., }
$$


therefore $g$ is a constant a.e. and $\{\phi, \hat{\phi}\}$ is Pauli unique. In a similar manner we can prove

THEOREM 7. Let $A=p^{2}+w\left(q_{1}, \ldots, q_{n}\right), p^{2}=\sum_{i=1}^{n} p_{i}^{2}$, be a real operator. Then any real state $\left\{\phi\left(q_{1}, \ldots, q_{n}\right), \hat{\phi}\left(p_{1}, \ldots, p_{n}\right)\right\}$ with real representative $\phi\left(q_{1}, \ldots, q_{n}\right)$ is Pauli unique if $E_{\phi}(A)<\infty$.

Proof. The proof is the same as for Theorem 3. A similar theorem holds if $A=q^{2}+u\left(p_{1}, \ldots, p_{n}\right)$, and the state has real representative $\hat{\phi}(p)$ with $E_{\phi}(A)<\infty$.

Again we get the important corollary,

COROLLARY. If $H=p^{2} / 2 m+V(q)$ is a motion-reversal invariant (real) Hamiltonian operator, then every bound state of $H$ is Pauli unique.

Proof. We again note that any bound state of $H$ has a real representative $\phi(q)$ and use Theorem 7.

The complex Gaussian wave functions,

$$
\prod_{j=1}^{n}\left(2 \pi \gamma_{j}\right)^{-1} \exp \left\{-\left(q_{j}-\beta_{j} I\right)^{2} / 4 \gamma_{j}+i \alpha_{j} q_{j}\right\}
$$

are Pauli unique. The proof of this needs the tensor product decomposition of $L^{2}\left(\mathbf{R}^{n}\right)$.

\section{Conclusions and general remarks}

A physical meaning for the Pauli problem is obtained if we accept that a quantum mechanical state results from the application of a certain state preparation procedure to a certain physical system [5], [2]. In the Pauli problem we try to determine the particular state that was prepared by that procedure by determining the momentum and position probability distributions of the prepared state. This is possible if we assume that the preparation procedure can be repeated an unlimited number of times so that we obtain the same state on each occasion and thereby can select subsets of identically prepared states on which the different determinative measurements can be carried out to any desired degree of accuracy.

With this interpretation our results show that in general it is not possible to determine uniquely the prepared state by merely determining the momentum and position probability distributions. In fact there are families of dense subsets of states that are not Pauli unique and hence any state can be arbitrarily well approximated, in norm, by a non-unique state. On the other hand, there are states that are uniquely determined once the expectation values of a finite number of 
observables $\left\{f_{i}(p)+g_{i}(q)\right\}_{i=1}^{N}$ are known. In particular it is interesting that the bound states of any semi-bounded Hamiltonian of the form $\left(p^{2} / 2 m\right)+V(q)$ are uniquely determined by the Pauli data. More specifically the energy eigenstates of atomic systems are uniquely determined by their momentum and position distributions, if we ignore any effects of spin. Of course this is somewhat artificial because the eigenstates of an atomic system will not be uniquely determined, in general, from their momentum and position probability distributions. This raises a difficulty in those theories of non-relativistic scattering in which the in and out scattering states are assumed to be determined by their energy and momentum probability distributions (see, for example [5]).

If we take the state of a quantum mechanical system to be defined by the position and momentum probability distributions, by analogy with the definition of state in classical mechanics in which the state is determined by the position and momentum of every particle in the classical system, then we have that the von Neumann definition of pure state coincides with the classical definition of state for bound states but not, in general, for scattering states. Expressed more precisely, if we define a pure $P Q$-state as the family of rays which satisfy the Pauli data then the pure $P Q$-state is equivalent to the pure von Neumann state for all bound states of a quantum mechanical system but they are not necessarily equivalent for scattering states.

If we enlarge the class of observables whose expectation values can be used to determine the state, it is reasonable that the class of states that can be uniquely determined will be enlarged. In many of the examples of non-uniqueness that we have produced it is easy to construct functions of $p$ and $q$ that distinguish between the Pauli partners. Moreover, there is a general method of determining the phase of a wave function. Let $\bar{p}(q) \Delta q$ be the conditional expectation of the momentum $p$ given that $q$ lies in $[q, q+\Delta q]$, then it has been shown by Moyal [3] that

$$
\bar{p}(q)=\frac{\partial \alpha(q)}{\partial q},
$$

where $\alpha(q)$ is the phase of the wave function $\phi(q)$. Therefore by carrying out a sequence of single slit experiments in which the location of the centre of the slit is varied, but everything else held fixed, we can in principle determine the phase $a(q)$ to within an additive constant, at least to any desired degree of approximation.

This interpretation of the Pauli problem leads to the following, more general, analysis. Suppose that we have prepared a state of a given system in the way described above and we now wish to determine the state by performing a series of determinative measurements of certain physical observables. Then there are two questions that naturally arise:

(1) What is the smallest collection of physical observables the determinative measurement of which determines the experimentally prepared state uniquely? 
(2) Given a subset of physical observables, which states are uniquely determined by the determinative measurements of these subsets?

The answers to these questions depends upon what are taken to be physical observables. In this work we have implicitly assumed that the physical observables are the kinematical observables, position, momentum, angular momentum including spin, as well as the energy and any superselected observables that may appear in the description of the system. For the system of a single one-dimensional particle the physical observables are $p, q$ but there is no angular momentum. In higher dimensions we would have to consider also angular momentum.

Finally, we have not discussed the multiplicity question; how many Pauli partners can a state have? In order to answer this question we would need to know what $\phi \in L^{2}(\mathbf{R})$ can be simultaneously an eigenfunction for two self-adjoint operators $A(p, q)$ and $B(p, q)$ that are functionally independent. This question requires more structure on the class of permissible self-adjoint operators than we have given here. What we can say is that if $\phi$ is an eigenfunction of a semi-bounded self-adjoint operator of the form $A(p, q)=f(p)+g(q)$, then it cannot be an eigenfunction of a self-adjoint operator $B(p, q)$ such that $e^{i B(p, q)}=e^{i F(p)} e^{i G(q)}$ for some pair of real measurable functions $F$ and $G$. It should be noted that this is a question specifically dependent on the fact that we are using differential operators, as simple examples in finite matrices will easily show.

\section{Acknowledgements}

One of us (J. V. C.) gratefully acknowledges the support of an A.R.G.C. Research Fellowship at the commencement of this research.

\section{Appendix}

The results that are needed to construct the non-unique states of Example 2, Section 3 , can be readily obtained from a consideration of the faithful representation of $S L(2, \mathbf{R})$ as uni-modular $2 \times 2$ matrices. It is sufficient to give the representation of the generators $J_{+}, J_{-}, J_{3}$ of the Lie algebra of $S L(2, \mathbf{R})$. These generators satisfy

$$
\left[J_{3}, J_{+}\right]=J_{+},\left[J_{3}, J_{-}\right]=-J_{-},\left[J_{+}, J_{-}\right]=2 J_{3}
$$

and all other commutators vanish. We take

$$
J_{+}=\left(\begin{array}{cc}
0 & 1 \\
0 & 0
\end{array}\right), \quad J_{-}=\left(\begin{array}{cc}
0 & 0 \\
1 & 0
\end{array}\right) \text { and } J=\left(\begin{array}{cc}
\frac{1}{2} & 0 \\
0 & -\frac{1}{2}
\end{array}\right) \text {. }
$$


For any real numbers $a, b, c, d$ the following members of the group $S L(2, \mathbf{R})$ are singled out,

$$
\left.\begin{array}{rl}
g_{1}(a) & =\exp a J_{+}, \\
g_{2}(b) & =\exp b J_{-} \\
g_{3}(c \cdot d) & =\exp \left(c J_{+}+d J_{-}\right) .
\end{array}\right\}
$$

The following facts can be verified by direct calculations using the given faithfu! representation.

LemMa A1. For strictly positive real numbers $K$ and $m$ the equation

$$
g_{1}(s) g_{2}(t)=g_{3}\left(K,-\frac{1}{m}\right)
$$

holds only when there exists an integer $n$ such that $K=4 \pi^{2} m n^{2}$, and in this case $s=t=0$.

In other words, the equation (A2) only has the trivial solution in the group $S L(2, \mathbf{R})$, e.e $=e$, where $e$ is the identity element of $S L(2, \mathbf{R})$, because for all integers $n, g_{3}\left(4 \pi^{2} m n^{2},-1 / m\right)=e$.

Let $K$ and $m$ be strictly positive real numbers as before and consider the constants $\alpha=) K / m)^{\frac{1}{2}}, \beta=(K m)^{\frac{1}{2}}$; if $\alpha \neq n \pi$ for any integer $n$, we define

$$
\left.\begin{array}{l}
s=2 \beta(1-\cos \alpha) / \sin \alpha \\
t=\sin \alpha / \alpha
\end{array}\right\}
$$

By direct calculation we get

LEMMA A2. Let $s, t$ be defined as in (A3), $\alpha \neq n \pi$, then the following identity holds in $S L(2, \mathbf{R})$,

$$
g_{1}\left(\frac{1}{2} s\right) g_{3}\left(K,-\frac{1}{m}\right) g_{1}\left(-\frac{1}{2} s\right)=g_{1}(s) g_{2}(-t) .
$$

As a consequence of these relations in $S L(2, \mathbf{R})$ we have that for any faithful representation $\pi$ of $S L(2, \mathbf{R})$,

$$
\pi\left(g_{1}(a)\right) \pi\left(g_{2}(-b)\right) \neq \pi\left(g_{3}\left(K,-\frac{1}{m}\right)\right)
$$

unless $a=0, b=0$ and $K=4 \pi^{2} m n^{2}$, that is $\alpha=2 \pi n$, for some integer $n$; and

$$
\pi\left(g_{1}\left(\frac{s}{2}\right)\right) \pi\left(g_{3}\left(K,-\frac{1}{m}\right)\right) \pi\left(g_{1}\left(-\frac{s}{2}\right)\right)=\pi\left(g_{1}(s)\right) \pi\left(g_{2}(-t)\right)
$$


holds for arbitrary real positive $K$ and $m$, with $\alpha=(K / m)^{\frac{1}{\mathbf{t}}} \neq n$ for any integer $n$, and $s$ and $t$ given by equations (A3).

In particular, consider the faithful unitary representation $U(g)$ of $S L(2, \mathbf{R})$ on $L^{2}(\mathbf{R})$ in which the generators of the Lie algebra

$$
J_{+}=\frac{1}{2} i q^{2}, \quad J_{-}=-\frac{1}{2} i p^{2}, \quad J_{3}=\frac{1}{4} i(q p+p q)
$$

are unbounded skew-adjoint operators with dense common domain $S(\mathbf{R})$. The equation (A6) takes the form

$$
e^{i s q^{2} / 4} \exp \left\{i\left(\frac{p^{2}}{2 m}+\frac{K}{2} q^{2}\right)\right\} e^{-i s q^{2} / 4}=e^{i s q^{2} / 2} e^{-i t p^{2} / 2} .
$$

But $\left(p^{2} / 2 m\right)+(K / 2) q^{2}=H(m, K)$, the harmonic oscillator Hamiltonian for mass $m$ and constant $K$, and hence we have shown that for given $K, m$ with $(K . m)^{\frac{1}{2}} \neq n \pi$, and $s$ and $t$ given by (A3)

$$
e^{i H(m, K)}=e^{i s q^{2} / 4} e^{-i t p^{2} / 2} e^{i s q^{2} / 4},
$$

that is

$$
e^{i H(m, K)}=e^{-i s q^{2} / 4}\left(e^{i s q^{2} / 2} e^{-i l p^{2} / 2}\right) e^{i s q^{2} / 4}
$$

and $e^{i H(m, B)}$ is unitarily equivalent to $e^{i F(q)} e^{i G(p)}$ with $F(q)=s q^{2} / 2, G(p)=-t p^{2} / 2$.

\section{REFERENCES}

[1] G. Hellwig, Differential operators of mathematical physics (Addison-Wesley Pub. Co., Reading, Palo Alto, London, Don Mills, 1964), Ch. 6, Sect. 1.

[2] J. M. Jauch, Foundations of quantum mechanics (Addison-Wesley Pub. Co., Reading, Menlo Park, London, Don Mills, 1968).

[3] J. E. Moyal, Proc. Cambridge Phil. Soc. 45 (1949), 99.

[4] W. Pauli, "Die allgemeinen Prinzipien der Wellenmechanik", in Handbuch der Physik (Springer-Verlag, Berlin, 1958), Vol. V, p. 17.

[5] E. Prugovečki, Quantum mechanics in Hilbert space (Academic Press, New York and London, 1971).

School of Mathematics and Physics

Macquarie University

North Ryde, N. S. W. 2113

Australia

and

Department of Mathematical Physics

University of Adelaide

Adelaide

South Australia 5001 